\title{
Scanning Electron Microscopy: Extrapolation of 3D Data from SEM Micrographs
}

\author{
Simonas KAREIVA ${ }^{1}$, Algirdas SELSKIS ${ }^{2}$, Feliksas IVANAUSKAS ${ }^{3}$, \\ Simas ŠAKIRZANOVAS ${ }^{1,2}$, Aivaras KAREIVA ${ }^{1 *}$ \\ ${ }^{1}$ Faculty of Chemistry, Vilnius University, Naugarduko 24, LT-03225 Vilnius, Lithuania \\ ${ }^{2}$ Institute of Chemistry, Center for Physical Sciences and Technology, A. Gostauto 9, LT-01108 Vilnius, Lithuania \\ ${ }^{3}$ Faculty of Mathematics and Informatics, Vilnius University, Naugarduko 24, LT-03225 Vilnius, Lithuania \\ cross $^{\text {ref }}$ http://dx.doi.org/10.5755/j01.ms.21.4.11101
}

Received 27 March 2015; accepted 17 May 2015

\begin{abstract}
In this manuscript we suggest a three-dimensional reconstruction technique to fully characterize structural performance of solid materials. The described technique extrapolates, measures and interprets the 3-dimensional data which is extracted from SEM images, obtained from different angles. Further, finer results were achieved by extrapolating of spatial data from three or more sample images using visual reconstruction software applications. Gold particles, silicon wafers and dendrites were selected as model materials for the spatial 3D surface reconstruction. For comparison and proof-of-concept, stereoscopy technique was also included into the research.

Keywords: SEM, 3D images, spatial reconstruction, stereoscopic analysis.
\end{abstract}

\section{INTRODUCTION}

The process of morphological characterization of a specimen using scanning electron microscopy (SEM) generally produces a magnified specimen image which is built out of the ejected electron beam raster scan data and the initial position of the beam. The scanning electron microscope provides large depth-of-focus and highcontrast images at a large variability in magnification. Nevertheless, the resulting image is literally a twodimensional intensity distribution array and usually does not carry any additional specimen-related metadata, except for the observational circumstances. To extract spatial data from two-dimensional images can be difficult. Thus, to achieve this the specimen must be handled in a special way and afterwards the produced micrographs must be digitally processed. However, this characterization method can not be used for many specific fundamental and applied investigations, for example, characterization of crack formation within cement paste under subcooling temperature or to study the fracture mechanisms in different materials, and many others [ $1-7]$.

In materials science, for three-dimensional reconstruction of microstructures various techniques, such as X-ray diffraction contrast tomography or X-ray tomography and mechanical sectioning have been applied successfully for decades [8-10]. X-ray computed microtomography, sometimes combined with micro-X-ray fluorescence technique is often used in medicine and material characterization as a non-invasive method for visualizing three-dimensional structure of various objects. Electron tomography has also gained a good reputation as a reliable nanoscale 3D-reconstruction technique in materials science. Its application relies on the transmission electron microscope (TEM) operating as a projection tool, which delivers an image intensity distribution proportional

\footnotetext{
* Corresponding author: Tel +370 52193110 .

E-mail address: aivaras.kareiva@chf.vu.lt (A. Kareiva)
}

to the projected density of material [11-13]. It was demonstrated, that optical coherence tomography allows rapid evaluation of coating surface morphological properties and three-dimensional images could be reconstructed [14].

The unique capabilities of ultrashort pulse femtosecond lasers have been integrated with a focused ion beam platform to create a new system for rapid 3D materials analysis [15]. Consequently, the combination of integrated focused ion beam-scanning electron microscope (FIB-SEM) serial sectioning and imaging techniques with image analysis was recently used for quantitative threedimensional characterization of different materials, such as catalysts [16], SOFC [17], pigments [18], cathode for lithium-ion cells [19].

Electron microscopy has made a great impact on structure determination, however, only TEM showed a strong potential for structural studies of nano-crystals, interfaces, phase boundaries and their surfaces [20, 21]. On the other hand, scanning electron microscopy is one of the most powerful techniques for studying fine structures of different surfaces. For example, SEM stereoscopic technique was used to determine the three-dimensional surface structures of volcanic ash particles [22] or pipettes [23], or even surface properties of nanostructures [24]. It is very well known, that stereoscopy simulates human vision by combining two pictures of a specimen from two slightly different angles. The depth of the produced stereoscopic image depends on the chosen angle where 2.5, 5 or 10 degrees [25] is usually enough to produce a sufficient depth perception to the viewer. Stereoscopic images are usually called anaglyphs [26,27]. The success of this technique highly depends on the microscope type and its instrumental precision. Stereo photography requires precise compucentric tilting of the observed sample, so that during the tilt, image focal points are preserved in the microscope viewport [28]. This is difficult to achieve for some SEM models, where the accuracy is linearly decreased, as the magnification increases. However, if the 
tilt is precisely compucentric, anaglyphs of magnification up to 50000 times can be easily produced. Otherwise, the SEM operator must manually apply the compensation of the shift that happens during the tilt.

The aim of this study is to extrapolate, measure and interpret 3-dimensional sample data out of the SEM images obtained from different angles. It is expected that during this study, the employed algorithms of visual reconstruction should be modified with respect to the difference of properties of an electron beam and visible light, and photon behaviour. In that case they could fit for the microscopic surface analysis. This paper also aims to uniquely address the issue of spatial reconstruction techniques of scanning electron microscope for sample surfaces and to provide recommendations and methods for applications of available spatial reconstruction software libraries. In this particular study, we investigate the method of structure-from-motion reconstruction combined with the manoeuvring capabilities and precision of modern scanning electron microscopes. The gold particles, dendrites and silicon wafers were selected as model materials for the spatial 3D surface reconstruction. For the comparison, SEM stereoscopic technique was also used to determine the three-dimensional surface structures.

\section{EXPERIMENTAL}

As we already mentioned, the stereoscopy simulates human vision by combining two pictures of a specimen from two slightly different angles (see Fig. 1). This approach was used for the proof-of-concept reconstruction of specimen spatial data. Combination of two SEM images into an anaglyph can be easily done with any freely available software, such as AnaMaker [28]. Subsequently, widely available LC-3D shutter glasses can be used for viewing the $3 \mathrm{D}$ images. Several spatial data extrapolation techniques are available when the initial input consists of only two images $[29,30]$. However, to achieve finer results, extrapolation of spatial data was done from three or more sample images using visual reconstruction software applications.

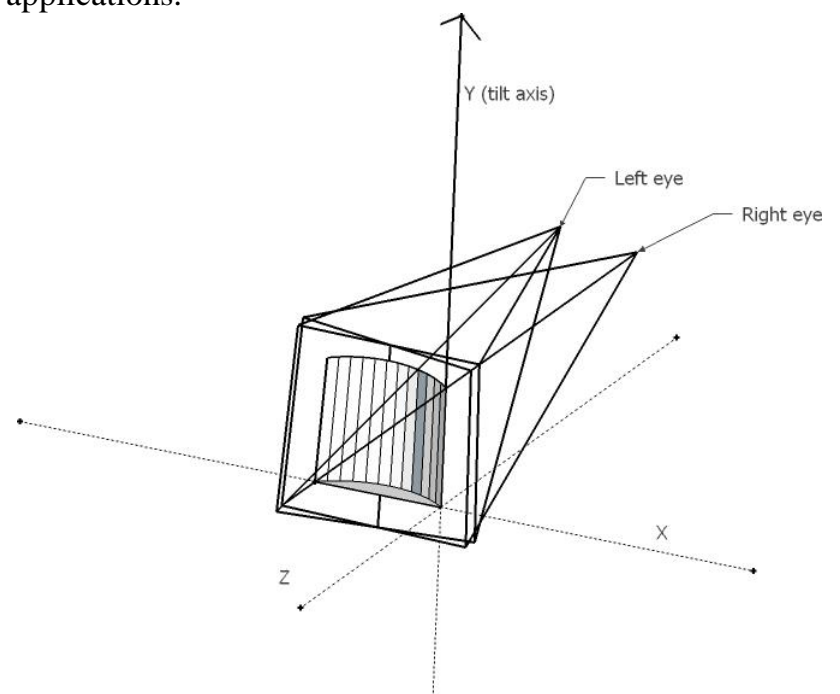

Fig. 1. Position of stereoscopy viewports at the SEM sample

Scanning electron microscopy (SEM) micrographs of investigated samples were obtained by a FE-SEM Hitachi SU-70 scanning electron microscope. SEM images for the reconstruction were obtained from Helios NanoLab 650 model microscope, manufactured by FEI. Analysis of conductive samples was performed with nominal beam voltage of $5 \mathrm{kV}$, which allowed the widest distribution of scattered-beam intensities and, thus, resulted in best image contrasts.

Tilting manoeuvres of these samples on the SEM table were performed in steps from 5 to 15 degrees, up to a maximum tilt of 45 degrees. Additionally, most experiments involved tilting both on $y$ and $x$ axes, so that more sample details were revealed which add up to the precision of the reconstruction process in terms of both detail and proportion. Generally, it is obvious that if the reconstruction relies on viewports residing on $N$ dimensional axes of tilting, the resulting spatial model is disproportional in $N+2$ dimensions.

The materials investigated in this study were gold particles (Agar Scientific Ltd.), dendrites (Agar Scientific Ltd.) and silicon wafers (JSC Precizika-MET SC, Lithuania). The gold calibration specimens on carbon have been specifically produced for the assessment of highresolution capabilities of SEM. Medium resolution aluminium-tungsten dendrites were also used in these investigations. The various spacings created by the dendritic structure of this specimen are suitable for performing point resolution tests and the topographical arrangement of the dendrites for a grey level test. The p-type mono-crystalline Si (100) wafers with thickness of $200 \mu \mathrm{m}$ and resistivity about $1-5 \Omega \cdot \mathrm{cm}$ were used for samples preparation. A texturization of wafers was performed in $\mathrm{KOH}-\mathrm{IPA}$ (isopropyl alcohol) solution at a temperature $85^{\circ} \mathrm{C}$ for $70 \mathrm{~min}$. Such a long texturization time was chosen in order to etch completely saw-damage from the surface and avoid additional saw-damage removal process [31]. The textured wafers were cleaned in $\mathrm{HCl}: \mathrm{H}_{2} \mathrm{O}_{2}: \mathrm{H}_{2} \mathrm{O}$ mixture at $65-75^{\circ} \mathrm{C}$ for $15 \mathrm{~min}$ and then wafers surfaces $\mathrm{H}$-terminated in $\mathrm{HF} / \mathrm{DI} \mathrm{H}_{2} \mathrm{O}$ for $1 \mathrm{~min}$ in order to remove the thin oxide layer and some fraction of ionic contaminants.

\section{RESULTS AND DISCUSSION}

Fig. 2 shows SEM image of gold particles investigated in this study. As seen from SEM image, the gold specimen consists of plate-like particles. Additionally, the microstructure of the gold sample is also composed by a number of different size $(0.2-3 \mu \mathrm{m})$ grains. An example of anaglyph created with AnaMaker is shown in Figure 3. In this case, to analyze the surface microstructure the 3D glasses should be used. The stereoscopic SEM image of $\mathrm{Au}$ particles reveals the formation of plate-like particles along with much smaller spherical particles. It is obvious that surface roughness of the Au sample could be estimated from 3D SEM image. However, this technique requires that images for both eyes should be taken with some precision, at least to keep the analysed sample in the centre.

Another technique for recovering spatial data from the SEM pictures (structure-from-motion) is described below. Currently proposed solution is the VisualSFM software, which is applied for spatial reconstruction using structure from sample motion. 


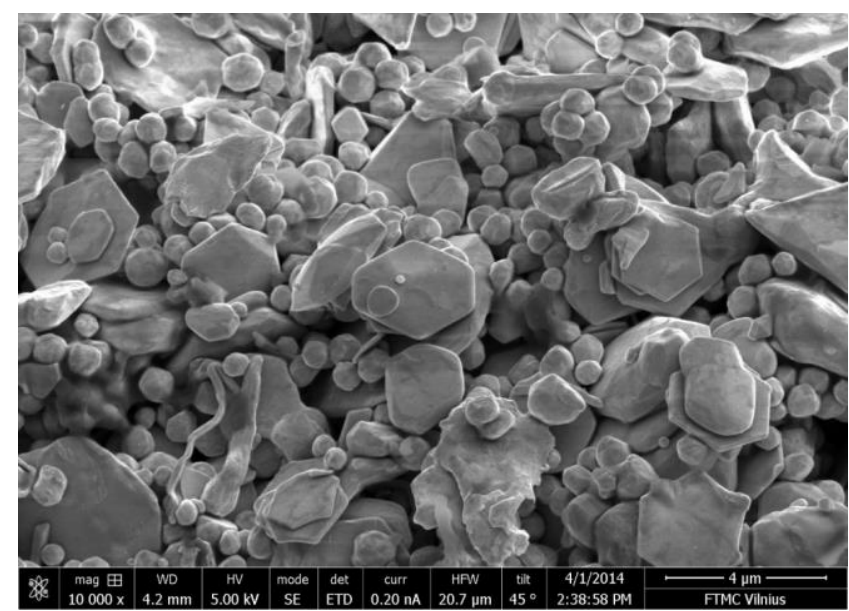

Fig. 2. SEM micrograph of Au sample

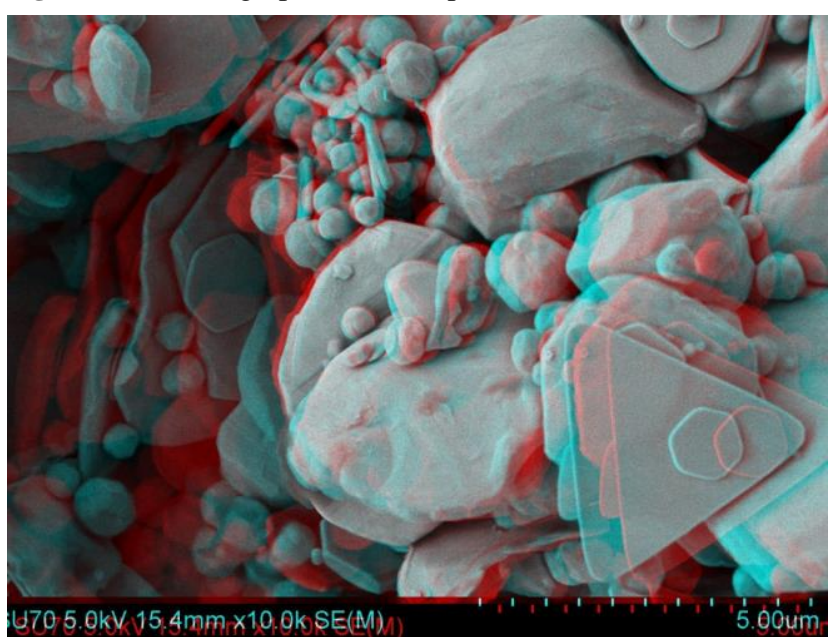

Fig. 3. A stereoscopic SEM image of Au particles

As seen in Fig. 4, this process usually requires additional viewports. The input images are analyzed by VisualSFM for matching points and the camera angle is guessed for each image. After the viewport intersections have been identified, the software library continues to dense reconstruction process. This produces results similar to those displayed in Fig. 5. Any number of additional viewports can be added to VisualSFM software. Based on this input, a surface is reconstructed where the matching points intersect and a colour value is assigned. The 3D image of the same Au particles reconstructed from 12 SEM micrographs using spatial reconstruction is presented in Fig. 5. The software library produces a cloud of points, which has to be processed externally. Freely available spatial data processing software, such as MeshLab can be used to join the point cloud to a mesh and, as a second step of reconstruction, apply surface properties to the mesh polygons. The resulting spatial data indicates the development of weaker areas and a higher degree of heterogeneity in analysed local microstructure. As shown in Fig. 5, a dense microstructure that is composed of angular particles was observed in gold sample. Unique $\mathrm{Au}$ surface details make it easier to detect matches in several images. Therefore, this specimen produced the best results among other samples.

The Au sample was also least affected by the shadow motion induced by sample tilt when the sample in the SEM is tilted without moving the electron beam source.
Moreover, further statistical analysis could be performed on the image analysis data to determine particle size distribution and particle connectivity.

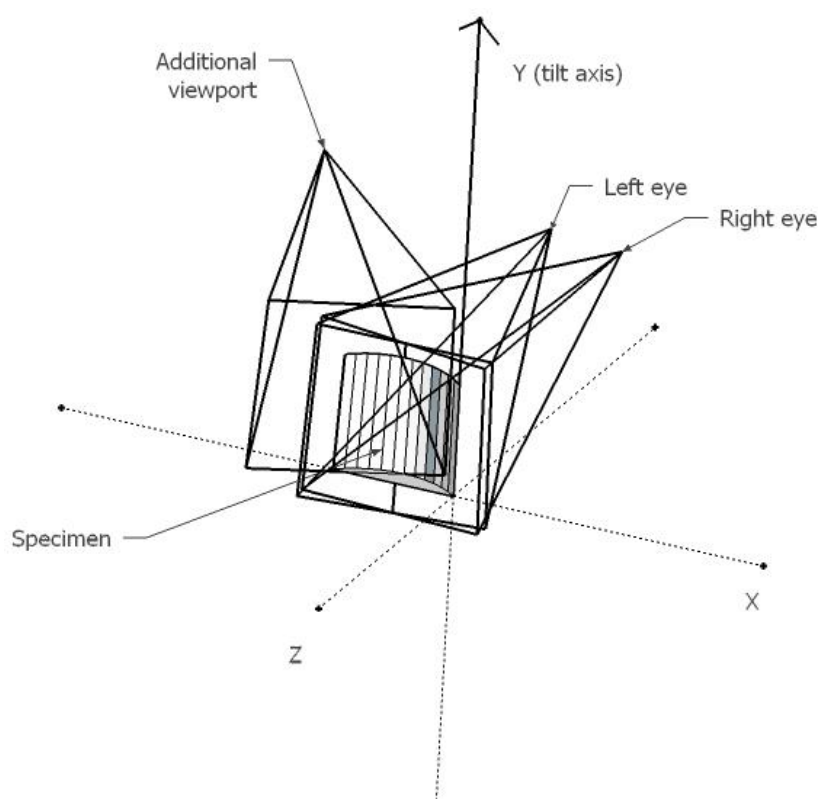

Fig. 4. Positions of additional viewports could be added to VisualSFM software
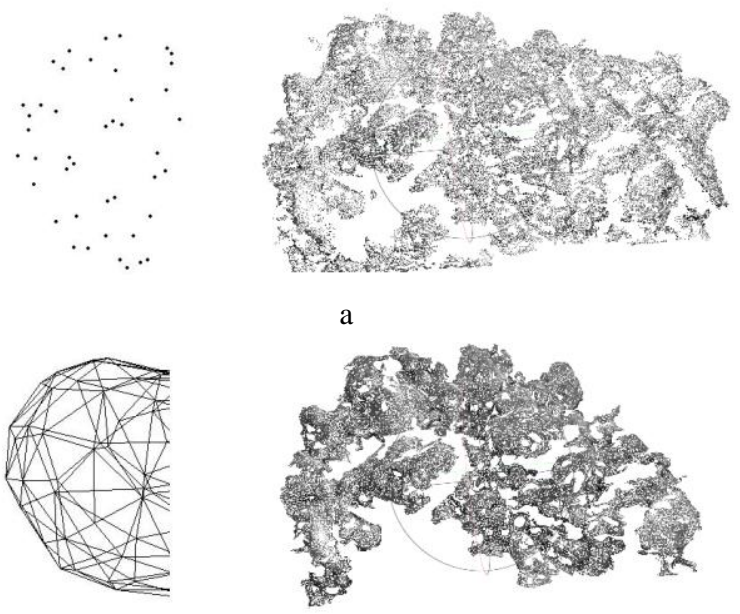

b
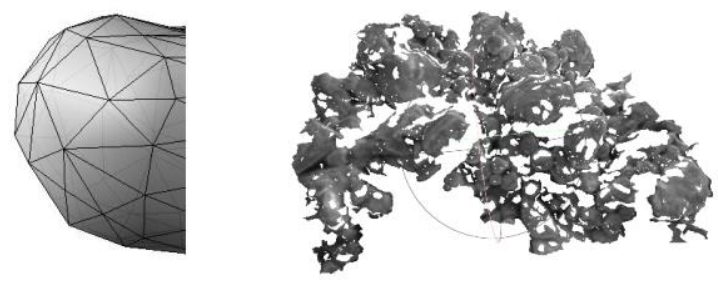

c

Fig. 5. 3D gold surface reconstruction steps, from point cloud (a), to mesh (b) and surface (c) on the right side and schematic explanation of steps on the left side

The "random pyramid" surface of silicon crystals was also taken as a sample for digital reconstruction. The SEM micrograph and an anaglyph of silicon wafer surface created with AnaMaker are shown in Fig. 6.

The sample shows particles with octahedral growth according to the model of compact crystalline planes 
where size is controlled by diffusion and with quite high crystal perfection [2].

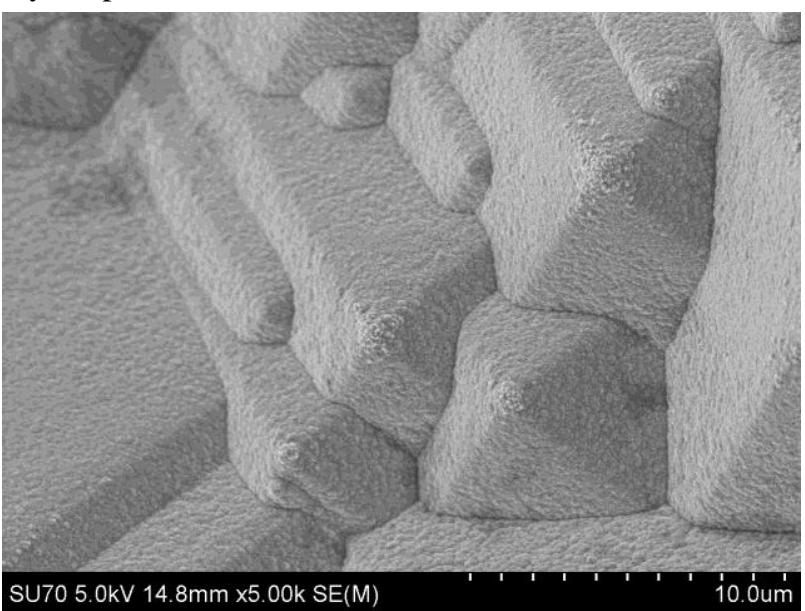

a

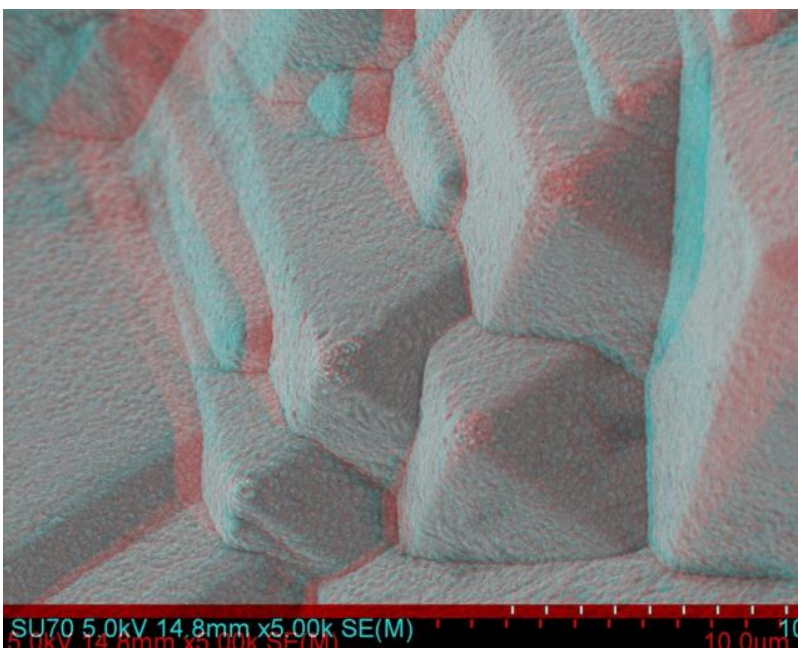

b

Fig. 6. $a-S E M$ micrograph of $\mathrm{Si}$ surface; $b$ - stereogram of $\mathrm{Si}$ surface

Besides, the micrometric silicon crystals show a high agglomeration degree. To reduce the surface reconstruction input data to the minimum, a total three images were obtained from viewports placed on xz-plane (as displayed in Fig. 7). Nevertheless, the reconstruction produced a distorted, but accurate point cloud, which closely resembled the actual surface. The mathematical expression of the reconstruction accuracy can be established with SEM calibration samples or material with known surface properties and the aforementioned proof is not included within the scope of this manuscript. Progressive new aspects of morphology could be deduced using 3D reconstructed images. The image of the silicon sample shows that individual particles seem to be square-like crystals. Moreover, the micrograph also shows lack of grain growth and undeveloped microstructure, probably, due to the partial melting or instability of the specimen under high calcination temperature [32].

Finally, the digital surface reconstruction method allows studying microscopic arrangements in silicon, on surfaces and at interfaces.

The dendrite crystals were the last sample, which has been successfully reconstructed in this study.

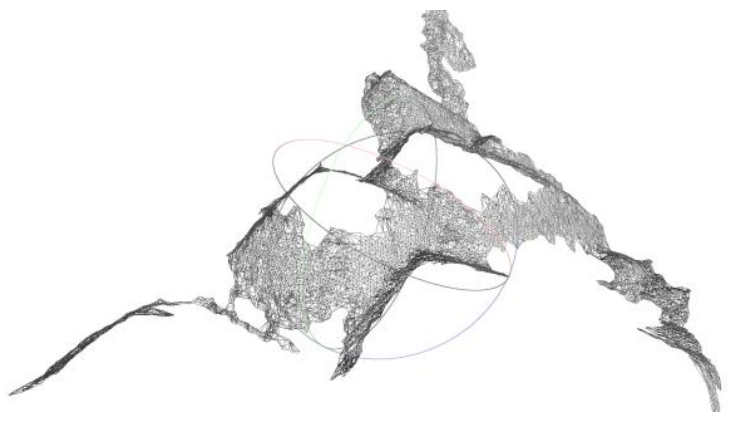

a

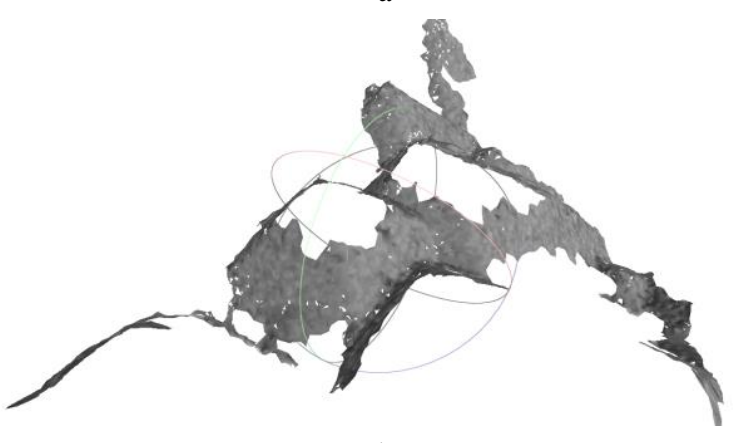

$\mathrm{b}$

Fig. 7. Digital reconstruction of Si surface from three images, xzplane views only, $17 \mathrm{k}$ faces, with steps from mesh (a) to surface (b)

The microstructure of sample consists of a clustered matrix, which is typical for the dendrites (Fig. 8). The specimen was scanned from 10 random angles, according to visual reconstruction requirements. The produced point cloud was of unknown spatial proportion and comparatively flat, but it closely resembled the crystal surface (Fig. 9). The suspected reason for the flatness was a very little change in angle during the initial shoot. Nevertheless, additional measurements should be performed as part of this work to determine if the point cloud is approximately proportional on the xy and z-axes.

The overall quality of the results for the described reconstruction technique depends on several factors, such as image contrast, homogeneity, angle and shadow variation. Most influential is image contrast and focus, which allows the software to easily match points and edges in a pair of pictures. This works together with the quality of selected specimen surface area, which obviously has to include at least some clearly visible surface details. Next factor is the chosen angle between viewports, which must correspond to the flatness of the surface, i.e., for comparatively flat surfaces, bigger angles must be chosen, so that during the tilt, as many surface details as possible are uncovered. The best results are achieved when the sample is scanned by several, from 10 to 15 semi-random viewports, in steps of approximately 2.5 degrees. Using bigger angles usually discovers more surface details, but on the other hand, has some drawbacks.

As it was mentioned above, SEM tilting is not possible without movement of shadows, since the electron beam remains fixed with the sensor and only the specimen is moving. In the case when shadows cover some important details of surface, the reconstruction software may not be aware of it and, hence, misses to match the points and/or edges in a particular image pair. This implication reduces 
the quality of surface which is covered by the tilt shadows. This applies especially to the deep gaps, cracks and pores.

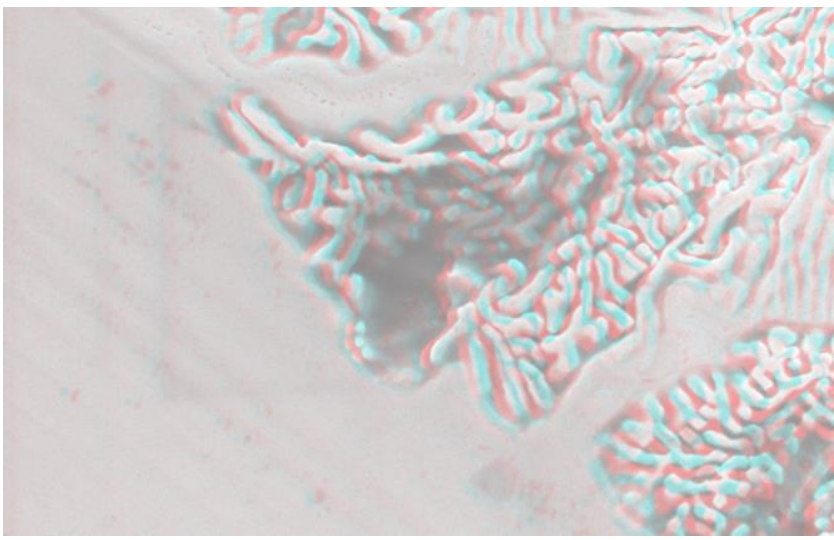

a

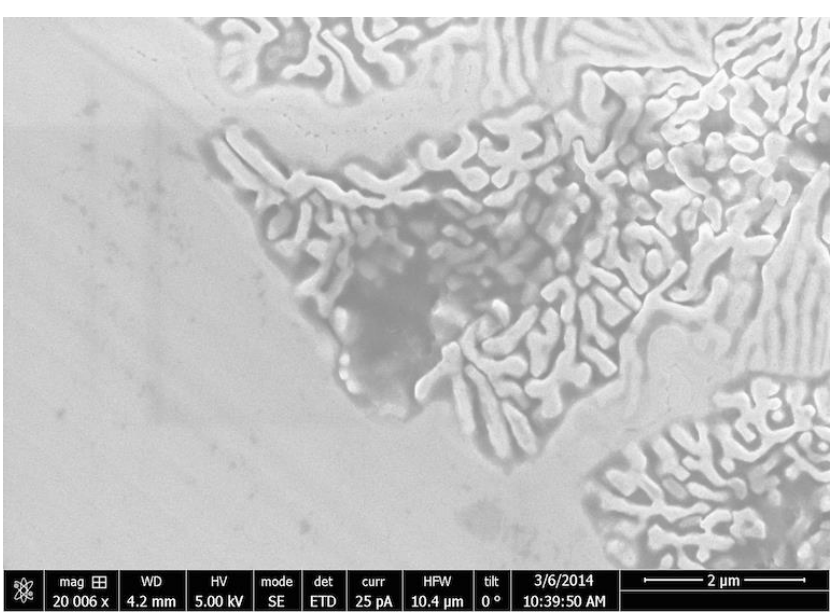

$\mathrm{b}$

Fig. 8. a-SEM micrograph of dendrites, b-stereogram of dendrites

The structural reconstruction produces a digital point cloud and each point retains an intensity value. For further processing of the point cloud, we use Ball pivoting algorithm which purpose is to detect and join nearest points into triangular faces so that a mesh and next a surface is produced.

In order to examine and exploit the capabilities of three-dimensional reconstruction, the 3D display and animation for this work has been implemented. This study suggests that size and shape of the true particles could be precisely determined. Grain boundaries, the interfaces between crystals, which are the principal microstructural defects in polycrystalline materials and consequently control many properties of engineering materials could be investigated using the proposed 3D reconstruction technique. Spatial reconstruction of SEM images will be invaluable method to investigate the microstructure and porosity of any materials and composites or cross sections of different thin layers [33-35]. The 3D reconstruction of sol-gel derived calcium hydroxyapatite samples, validation of the proposed reconstruction technique and application for quantitative microstructural analysis are under investigation. The information obtained will lead to the development of new structures with enhanced properties and functionalities in bulk bioceramic materials, nanoparticles and thin films.

\section{CONCLUSIONS}

New scanning electron microscopy method to obtain $3 \mathrm{D}$ structural information has been developed.

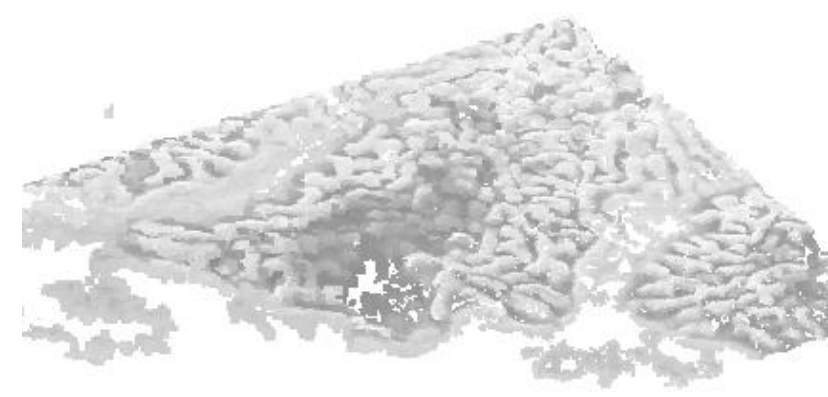

a

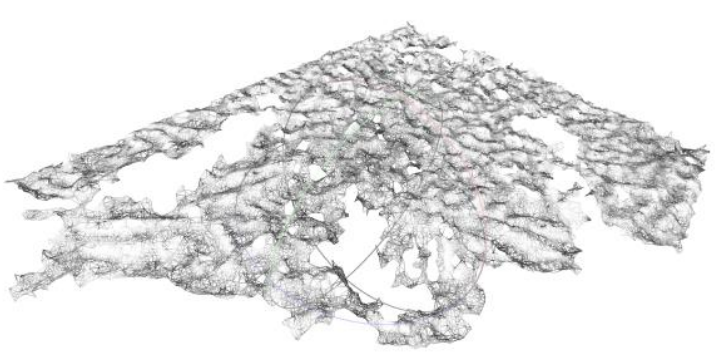

b

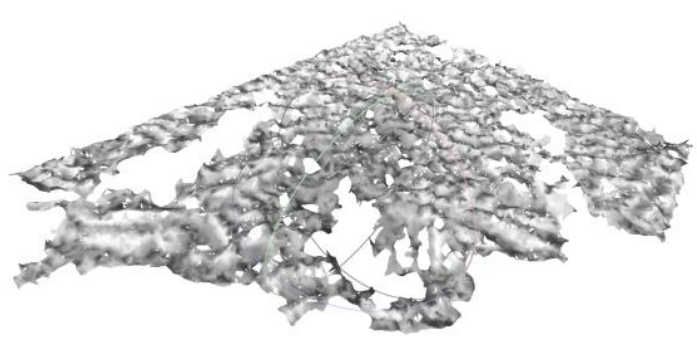

c

Fig. 9. An example of spatial reconstruction of dendrite crystals using several SEM images, with steps from point cloud (a), to mesh (b) and surface (c)

Three-dimensional reconstruction technique was employed to provide a deeper insight in the morphology of solid materials. The 3D sample data out of the SEM images obtained from different angles were extrapolated, measured and interpreted. The gold particles, silicon wafers and dendrites were selected as model materials for the spatial 3D surface reconstruction. The SEM image of $\mathrm{Au}$ particle indicated that gold consists of plate-like particles of $0.2-3 \mu \mathrm{m}$ in size. The 3D image of the same $\mathrm{Au}$ particles reconstructed from SEM micrographs using spatial reconstruction indicated development of grain boundaries in local microstructure. An anaglyph of silicon wafer surface created with AnaMaker showed the particles with octahedral growth with quite high crystal perfection. New aspects of morphology were deduced using 3D reconstructed images. The image of the silicon sample showed that individual particles seem to be square-like crystals with lack of grain growth and undeveloped microstructure. The microstructure of dendrite sample consisted of a clustered matrix. Further statistical analysis could be performed on the SEM image analysis data to 
determine particle size, particle size distribution and particle connectivity.

\section{Acknowledgement}

This research was partially funded by COST Action MP1202.

\section{REFERENCES}

1. Ng, K., Dai, Q.L. Numerical Investigation of Internal Frost Damage of Digital Cement Paste Samples With Cohesive Zone Modelling and SEM Microstructure Characterization Construction and Building Materials 2014:pp. 266-275. http://dx.doi.org/10.1016/j.conbuildmat.2013.09.025

2. Hallberg, H., Svendsen, B., Kayser, T., Ristinmaa, M. Microstructure Evolution during Dynamic Discontinuous Recrystallization in Particle-Containing $\mathrm{Cu}$ Computational Materials Science 84 2014: pp. 327-338. http://dx.doi.org/10.1016/j.commatsci.2013.12.021

3. Della Noce, R., Benedetti, A.V., Magnani, M., Passamani, E.C., Kumar, H., $\quad$ Cornejo, D.R., Ospina, C.A. Structural, Morphological and Magnetic Characterization of Electrodeposited Co-Fe-W Alloys Journal of Alloys and Compounds 2014 611: pp. $243-248$. http://dx.doi.org/10.1016/j.jallcom.2014.05.157

4. da Rocha, E.P., de Castro, J.A. Characterization and Three-Dimensional Reconstruction of Pores of SelfReducing Pellets Done By EAF Dust Materials Research - Ibero-American Journal of Materials 17 2014: pp. 47-55.

5. Echlin, M.P., Mottura, A., Wang, M., Mignone, P.J., Riley, D.P., $\quad$ Franks, G.V., Pollock, T.M. ThreeDimensional Characterization of The Permeability of W-Cu Composites Using A New „Tribeam“ Technique Acta Materialia 64 2014: pp. 307-315. http://dx.doi.org/10.1016/j.actamat.2013.10.043

6. Pirgazi, H., Ghodrat, S., Kestens, L.A.I. ThreeDimensional EBSD Characterization of Thermo-Mechanical Fatigue Crack Morphology in Compacted Graphite Iron Materials Characterization 90 2014: 13-20.

7. Kossivas, F., Angeli, S., Kafouris, D., Patrickios, C.S., Tzagarakis, V., Constantinides, C. MRI-Based Morphological Modeling, Synthesis and Characterization of Cardiac Tissue-Mimicking Materials Biomedical Materials 7 (3) 2012: pp. 035006-035016. http://dx.doi.org/10.1088/1748-6041/7/3/035006

8. Mobus, G., Inkson, B.J. Nanoscale Tomography in Materials Science Materials Today 10 2007: pp. 18-25.

9. Simonovski, I., Cizelj, L. Automatic Parallel Generation of Finite Element Meshes For Complex Spatial Structures Computational Materials Science 50 2011: pp. $1606-1618$.

10. Wan, Q., Zhao, H.D., Zou, C. Effect of Micro-Porosities on Fatigue Behavior In Aluminium Die Castings By 3D XRay Tomography Inspection ISIJ International 54 2014: pp. $511-515$.

http://dx.doi.org/10.2355/isijinternational.54.511

11. Mobus, G. Analytical Electron Tomography for Materials Science European Microscopy and Analysis 3 2003: pp. $15-17$.

12. Kawasaki, T., Takai, Y. Phase Reconstruction with Simultaneous Correction of Spherical and Astigmatic
Aberrations by Three-Dimensional Fourier Filtering Method Surface and Interface Analysis 35 2003: pp. 51-54. http://dx.doi.org/10.1002/sia.1492

13. Liu, Y.Z., Schreiber, D.K., Petford-Long, A.K., Gao, K.Z. Three-Dimensional Characterization of NearField Transducers by Electron Tomography Materials Characterization 72 2012: pp. 104-110. http://dx.doi.org/10.1016/j.matchar.2012.07.011

14. Koller, D.M., Hannesschlager, G., Leitner, M., Khinast, J.G. Non-Destructive Analysis of Tablet Coatings with Optical Coherence Tomography European Journal of Pharmaceutical Science 44 2011: pp. 142-148.

15. Echlin, M.P., Mottura, A., Torbet, C.J., Pollock, T.M. A New Tribeam System for Three-Dimensional Multimodal Materials Analysis Review of Scientific Instruments 83 (2) 2012: pp. $023701-023706$. http://dx.doi.org/10.1063/1.3680111

16. Singh, R., Akhgar, A.R., Sui, P.C., Lange, K.J., Djilali, N. Dual-Beam FIB/SEM Characterization, Statistical Reconstruction, And Pore Scale Modeling of A PEMFC Catalyst Layer Journal of Electrochemical Society 161 2014: pp. F415 - F424. http://dx.doi.org/10.1149/2.036404jes

17. Vivet, N., Chupin, S., $\quad$ Estrade, E., Richard, A., Bonnamy, S., Rochais, D., Bruneton, E. Effect of $\mathrm{Ni}$ Content in SOFC Ni-YSZ Cermets: A Three-Dimensional Study by FIB-SEM Tomography Journal of Power Sources 196 2011: pp. 9989-9997. http://dx.doi.org/10.1016/j.jpowsour.2011.07.010

18. Lin, J.C., Heeschen, W., Reffner, J., Hook, J. ThreeDimensional Characterization of Pigment Dispersion in Dried Paint Films Using Focused Ion Beam-Scanning Electron Microscopy Microscopy and Microanalysis 18 2012: pp. 266-271. http://dx.doi.org/10.1017/S143192761101244X

19. Ender, M., Joos, J., Carraro, T., Ivers-Tiffee, E. Quantitative Characterization of Lifepo 4 Cathodes Reconstructed by FIB/SEM Tomography Journal of Electrochemical Society 159 2012: pp. A972-A980. http://dx.doi.org/10.1149/2.033207jes

20. Syha, M., Trenkle, A., Lodermann, B., Graff, A., Ludwig, W., Weygand, D., Gumbsch, P. Validation of Three-Dimensional Diffraction Contrast Tomography Reconstructions by Means of Electron Backscatter Diffraction Characterization Journal of Applied Crystallography 46 2013: pp. 1145-1150.

21. Willhammar, T., Yun, Y.F., Zou, X.D. Structural Determination of Ordered Porous Solids by Electron Crystallography Advanced Functional Materials 24 2014: pp. $182-199$

22. Mills, O.P., Rose, W.I. Shape and Surface Area Measurements Using Scanning Electron Microscope StereoPair Images of Volcanic Ash Particles Geosphere 6 2010: pp. 805-811.

23. Malboubi, M., Gu, Y., Jiang, K. Characterization of Surface Properties of Glass Micropipettes Using SEM Stereoscopic Technique Microelectronic Engineering 88 2011: pp. 2666-2670. http://dx.doi.org/10.1016/j.mee.2011.02.029

24. Mohammadkhani, A., Malboubi, M., Anthony, C., Jiang, K. Characterization of Surface Properties of Ordered Nanostructures Using SEM Stereoscopic Technique Microelectronic Engineering 88 2011: pp. 2687-2690. http://dx.doi.org/10.1016/j.mee.2010.12.039 
25. Hortola, P. Using Digital Anaglyphy to Improve the Relief Effect of SEM Micrographs of Bloodstains Micron 40 2009: pp. 409-412.

26. Prathap, I., Anitha, R. Robust And Blind Watermarking Scheme For Three Dimensional Anaglyph Images Computers and Electrical Engineering 40 2014: pp. $51-58$.

http://dx.doi.org/10.1016/j.compeleceng.2013.11.005

27. Kempers, R., Ahem, P., Robinson, A.J., Lyons, A.M. Modeling The Compressive Deformation of Metal MicroTextured Thermal Interface Materials Using SEM Geometry Reconstruction Computers and Structures 92-93 2012: pp. 216-228.

28. Sekitani, T. 3D Software: Anaglyph Maker. http://www.stereoeye.jp/software/index_e.html 2001.

29. Demers, H., Poirier-Demers, N., Couture, A.R., Joly, D., Guilmain, M., De Jonge, N., Drouin, D. ThreeDimensional Electron Microscopy Simulation With The CASINO Monte Carlo Software Scanning 33 2011: pp. $135-146$.

30. Patane, G., Spagnuolo, M. Local Approximation of Scalar Functions on 3D Shapes and Volumetric Data Computers and Graphics-UK 36 2012: pp. 387-397.

31. Pranaitis, M., Jaraminė, L., Čyras, V., $\quad$ Selskis, A., Galdikas, A. Antireflective Structures On Silicon Surface Using Catalytic Nickel Nanoparticles Journal of Applied Physics 114 (16) 2013: pp. 163523-163527. http://dx.doi.org/10.1063/1.4827838

32. Min, J.W., $\quad$ Kalathil, A.K., $\quad$ Yim, C.J., $\quad$ Im, W.B. Morphological Effects on The Electrochemical Performance of Lithium-Rich Layered Oxide Cathodes, Prepared By Electrospinning Technique, For Lithium-Ion Battery Applications Materials Characterization 92 2014: pp. $118-126$. http://dx.doi.org/10.1016/j.matchar.2014.03.008

33. Klimavicius, V., Kareiva, A., Balevicius, V. Solid-State NMR Study of Hydroxyapatite Containing Amorphous Phosphate Phase and Nanostructured Hydroxyapatite: CutOff Averaging of CP-MAS Kinetics and Size Profiles of Spin Clusters Journal of Physical Chemistry C 118 2014: pp. 28914-28921.

34. Saarimaa, V., Manni, J., Kauppinen, E., Markkula, A., Juhanoja, J., Skrifvars, B.J. Cross-Sectional HighEsolution Microscopy of Thin Pretreatment Layers On Hot Dip Galvanized Steel Surface and Interface Analysis 46 2014: pp. 620-624.

35. Surmeneva, M.A., Surmenev, R.A., Nikonova, Y.A., Selezneva, I.I., Ivanova, A.A., Putlyaev, V.I., Prymak, O., Epple, M. Fabrication, Ultra-Structure Characterization and In Vitro Studiesof RF Magnetron Sputter Deposited NanoHydroxyapatite Thin Films For Biomedical Applications Applied Surface Science 317 2014: pp. 172-180. http://dx.doi.org/10.1016/j.apsusc.2014.08.104 